\title{
STRUCTURAL MONITORING IN THE "SANTA MARIA DE LA ASUNCIÓN" CATHEDRAL OF CHILPANCINGO, GUERRERO, MEXICO; THROUGH TOPOGEODESIC-PHOTOGRAMMETRIC SURVEYING AND AMBIENT VIBRATION. A METHODOLOGICAL PROPOSAL
}

\author{
SULPICIO SÁNCHEZ TIZAPA ${ }^{1 *}$, RODOLFO AURELIO FELICITO ${ }^{1}$, RENÉ \\ VÁZQUEZ JIMÉNEZ ${ }^{1}$, JOSÉ LUIS CARRANZA BELLO ${ }^{2}$ AND ROBERTO \\ ARROYO MATUS ${ }^{1}$ \\ ${ }^{1}$ Facultad de Ingeniería \\ Universidad Autónoma de Guerrero (UAGro) \\ Av. Lázaro Cárdenas s/n, Ciudad Universitaria, 39070 Chilpancingo, Guerrero \\ e-mail: sstizapa@uagro.mx,www.posgradoingenieria.uagro.mx (*corresponding author) \\ rodolfofelicito@uagro.mx,rvazquez@uagro.mx, arroyomatus@hotmail.com \\ ${ }^{2}$ Instituto Nacional de Estadística y Geografía (INEGI) \\ Av. Prolongación Baltazar R. Leyva Mancilla, Manzana 3, Lote 9, Primer Piso \\ Colonia Universal, 39080 Chilpancingo, Guerrero \\ luis.carranza61@yahoo.es,www.inegi.org.mx
}

Keywords: Historical Structure, Structural Health Monitoring, UAV, GPS.

\begin{abstract}
This paper presents a review of the state of the art of structural health monitoring using three inspection techniques and their application in the proposed methodology for the study of a historic structure located in a high seismic hazard area. The proposed approaches are a) use of an unmanned aerial vehicle (UAV), a reliable platform for data acquisition in photogrammetric inspection and surveillance work due to its simple handling and accessibility to human risk zones; b) Global Positioning System (GPS), which offers the capability of static or dynamic monitoring, using kinetic navigation (RTK), precise point positioning (PPP) or combination of both methods; c) ambient vibration (AV), an accelerometer test that determines the dynamic properties of a structure, of which values indicate the structural safety status. The building to be studied is the "Santa María de la Asunción" Cathedral of Chilpancingo in Mexico, which represents a great historical value because it hosted the First "Anahuac" Congress in 1813. The structure covers an area of $1056 \mathrm{sqm}$ and has a height of $33.31 \mathrm{~m}$. The construction began after 1902 and has been affected by several seismic events. Because of the earthquake of December 2011, cracks occurred in the basements and keystones of the arches of the two bell towers, severe damage to the right tower, and moderate to the left one. In the frontispiece, top of the arches, and in front of the central nave vault also appeared cracks. The reinforcement process is currently complete. The expected results are a matrix of distances among control points in the building, high-resolution ortho-images, and values of the dynamic structural properties. According to the architectural and structural characteristics, location, importance, and use of the structure, it is proposed to perform a monitoring every six months
\end{abstract}


or after an earthquake with a seismic magnitude greater than six and epicenter less than 60 $\mathrm{km}$. The comparison of the measurement parameters will indicate the structural health of the building.

\section{INTRODUCTION}

The damage reduces the mechanical properties of the structural elements, which adversely affect their current state. The implementation of a strategy for the identification of structural damage is known as structural health monitoring (SHM). It can be established in the short, medium, long term, or after extreme events occur. The goal of the evaluation is to provide, almost all in real-time, reliable data about the structural system conditions [1]. Currently, there are various inspection and structural monitoring techniques, among which stand out a) UAV [2], b) GPS [3], c) AV [4], d) laser scanning [5], among others.

\subsection{Unmanned aeriall vehicles}

Historically, the development of unmanned aerial vehicle systems and platforms were generated for military applications (inspection, surveillance, reconnaissance, and mapping of hostile areas). The early geomatics applications started about 35 years ago. UAVs are a valuable platform for data acquisition in inspection, surveillance, mapping, and 3D modeling. UAVs are considered a low-cost alternative compared to conventional manned aerial photogrammetry [6].
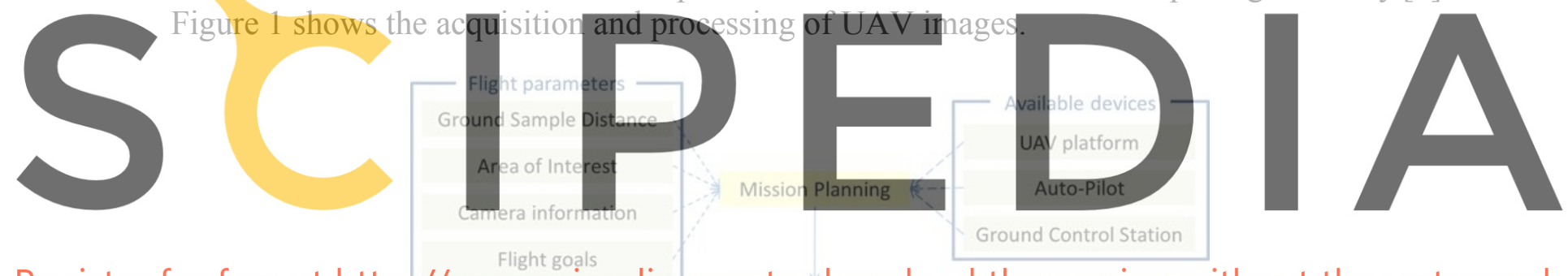

Register for free at https//WWW.scipedia.com to download the version without the watermark

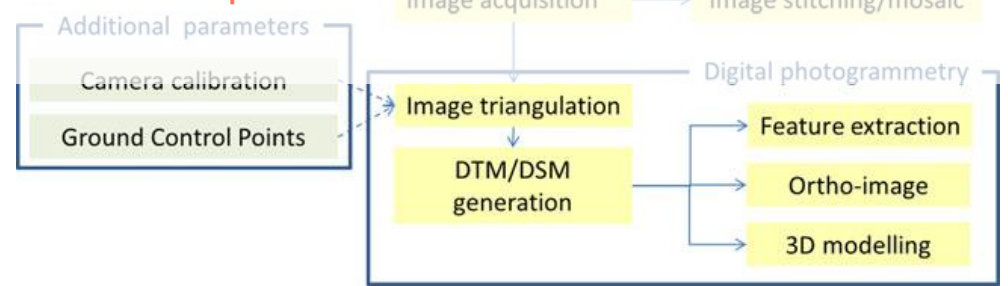

Figure 1: Typical acquisition and processing pipeline for UAV images [6]

Various researchers have made significant contributions to damage detection by using UAVs, i.e., the aim of a work conducted in South Dakota (USA) was to evaluate its effectiveness as a complementary tool for bridge damage quantification. In the study, a covered wooden arch bridge ( $88 \mathrm{~m}$ length and $7.9 \mathrm{~m}$ lane width) was selected. The protocol consisted of four stages: a) inspection, b) image assessment, c) damage quantification, d) damage classification according to the AASHTO standard. On inspection, the lengths and widths of cracks, thicknesses, and areas of rust spots were measured. Image quality parameters, including sharpness and entropy, were used to determine the quality of the obtained images. Then, pixelbased measurements and photogrammetry were obtained to quantify bridge damage and 
compared it with the field measurements. Next, the damage level classification was established based on current standards. The measurement difference shows a suitable accuracy with results within $3.5 \%, 7.9 \%$, and $14.9 \%$ for the length of the crack, the thickness, and the area of rust spots, respectively [7].

Another research conducted in Alaska (USA) on the Placer River Trail Bridge served as the evidence base for an inspection method with UAVs. The goal was to produce a threedimensional (3D) model using the captured images and a densely structured hierarchical algorithm based on motion. This aim guided the choice and equipment of the UAV as well as the planning of the flight mission. The resulting inspection integrates the technical characteristics of the UAV, the capture and analysis of data to supply a 3D model, which gives the inspection information allowing for defect monitoring. Results compared to models generated by laser scanning show that this method supplied more accurate information to identify defects or damages. Thus, data were obtained for decision-making by infrastructure managers [8].

For a long time, three-dimensional models of heritage buildings have employed image-based techniques. The technological development of optical sensors and dense matching algorithms complement the existing photogrammetric procedures in the study of historical buildings. An example is the studies of Rohan's palace and St-Pierre-le-Jeune Catholic Church (Strasbourg, France), where using aerial triangulation and a dense coincidence of UAVs Sensefly Albris and Phantom 3, the quality of the model was determined. Among the several used software (PhotoScan, Pix4D, MicMac, and PhotoModeler), MicMac obtained greater accuracy in both cases, it is a handy open-sourc

More recent research

patterns for the conserva

"Livorno" (Italy), a 3D
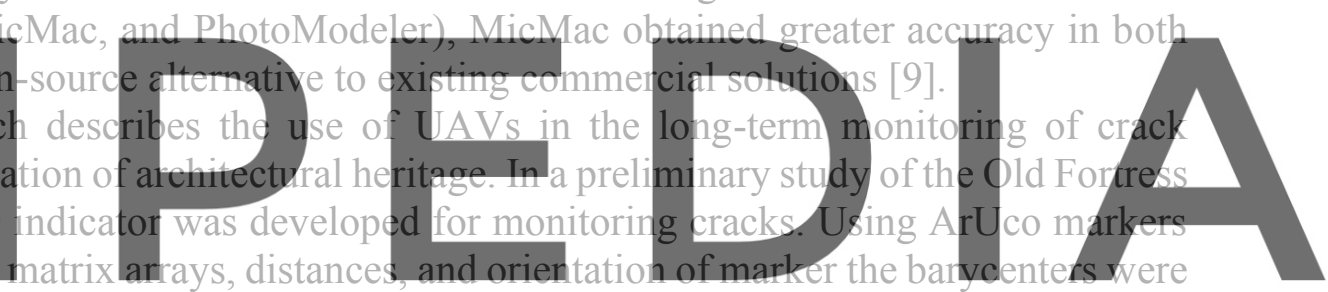

evaluated, obtaining a $5 \mathrm{~mm}$ projection error. The second method of simultaneous location and

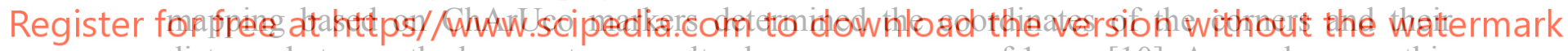
distance between the barycenters, results show an accuracy of $1 \mathrm{~mm}$ [10]. As can be seen, this second type of markers had greater accuracy

\subsection{Ambient vibration test}

The need for maintenance of structures has led to the development of experimental tests capable of determining the static and dynamic properties. In large structures, the most used structural health monitoring dynamic method is the Ambient Vibration (AV) test [11], which is measured by accelerometers. The human movement, traffic, building use, and machine vibrations excited the structures; its response can be measure by the Operational Modal Analysis.

Extensive research had been developed to obtain the dynamic properties and dynamic monitoring of the Mallorca cathedral [12], to know the global dynamic behavior of the structure and update the structural models. In the first case, modal parameters as natural frequencies, mode shapes, and damping ratios were obtained. Then, the mid-span points of the central and lateral arches of the structure were chosen to adapt the sensors to measure the ambient vibrations. The measure times for each setup test was greater or equal to 1000 times the 
fundamental period of the cathedral, around 15 minutes with a sampling frequency of $100 \mathrm{~Hz}$. Three tri-axial forced balanced accelerometers were used to carry out the tests. Four methods allowed to identify the dynamic properties: a) frequency domain decomposition, b) referencebased covariance-driven stochastic subspace identification, c) reference-based data-driven stochastic subspace identification, and d) poly-reference least-squares complex frequency domain identification. Eight natural frequencies were possible identified, the first was $1.15 \mathrm{~Hz}$, and the last was $2.6 \mathrm{~Hz}$. Respect to the damping ratios, the lowest was $0.44 \%$ (Mode 7 ), and the highest was 2.6\% (Mode 1); however, there were vast variations of different methods values. About the modes shape, the first mode has a predominant longitudinal over the transverse movement, the second and third modes show longitudinal movement, and the fourth showed transversal displacement, the torsion movement appeared in the eight mode shape. The wind blowing caused a transversal mode shape.

The dynamic monitoring system of Mallorca cathedral was developed with the tri-axial accelerometers above defined. Devices were exposed to the sunlight, rains, and out of the reach of anthropogenic action. The system worked for two periods: a) from December 17, 2010, to September 13, 2011, b) from May 18; 2012 to December 29, 2012, with an interruption from July 20 to September 4. The data were used to calculate the powers spectral density (PSD) for a signal length of half an hour with windows overlapped 33\%. The natural frequencies were determined using the picking method. The PSD calculation was carried out four times per day during the monitored period. The frequencies calculated every 6 hours is a detailed image of the daily change under different environmental conditions. In this case, the eight modes shape recorded during the AVT seismic events. Besides, their higher mass environmental paramete higher cost dynamic non
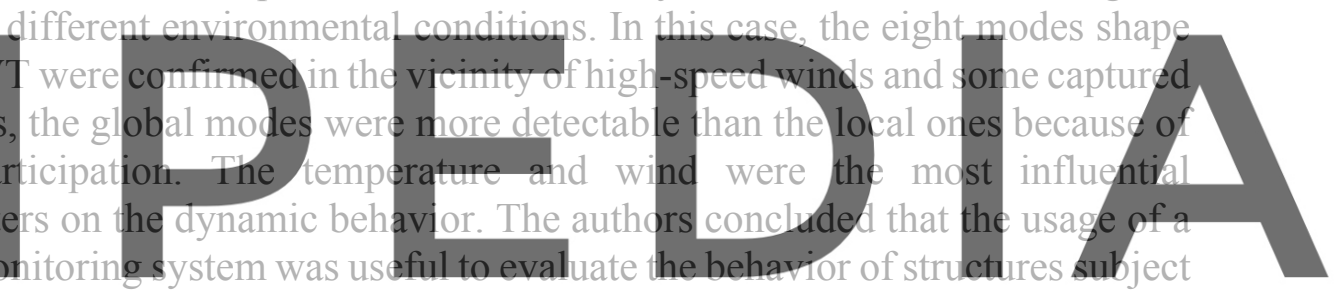

to low ground seismic accelerations. [12]

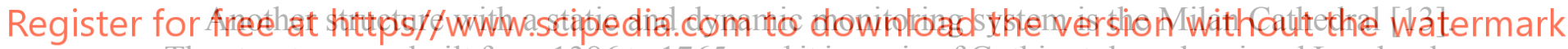
The structure was built from 1386 to 1765, and it is a mix of Gothic style and regional Lombardy style, where also neo-classic, neo-gothic. Even renaissance influences are present due to the prolonged period of construction. A distinctive characteristic is the connection of all adjacent piers using metallic elements to reduce the thrust on the slender lateral buttresses. The twomonitoring system measure different physical quantities, i. e.: a) quasi-static strains of selected tie-rods, b) indoor-outdoor environmental temperature, and relative humidity. Of the static monitoring system, eight modes were obtained by using referenced-based data-driven stochastic subspace identification (SSI-Cov). The first and second ones correspond to the sway motion of all instrumented columns in the North-South $\left(\mathrm{f}_{1}=1.58 \mathrm{~Hz}\right)$ and the Est-West $\left(\mathrm{f}_{2}=1.69\right.$ $\mathrm{Hz}$ ), respectively. The third and fourth modes recorded values of $\mathrm{f}_{3}=1.98 \mathrm{~Hz}$ and $\mathrm{f}_{4}=1.98 \mathrm{~Hz}$. Damping of the North-South modes had values from 1.79 to $4.14 \%$, and damping of the EstWest modes varies between 2.21 and 5.04\%. Almost all identified modes in the first hours of monitoring were detected in the subsequent months.

Dynamic monitoring results of Milan cathedral show that the outdoor temperature recorded variations from -2 to $+28{ }^{\circ} \mathrm{C}$ and indoor relative humidity from 38 to $64 \%$. About the modal frequencies, the lower five ones were greater than $98 \%$ of static monitoring values, while the coefficient of variation ranges between 0.34 and $1.10 \%$. All resonant frequencies increase 
linearly with decreased temperature. However, the relative humidity slightly affects only the first mode frequency. Finally, the mode shapes do not show visible fluctuations associated with the environmental effects, so that a suitable strategy of SHM should be based on the time invariance of those parameters. The period for two kinds of monitoring systems was from October 16, 2018, to February 13, 2019 [13].

\subsection{Precise positioning of points and ground control points with GPS}

The development of low-cost systems to collect spatial information near real-time eases the detection of the environment or the monitoring of disasters. At Cheng-Kung National University, Taiwan, a platform for obtaining spatial information based on fixed-wing unmanned aerial vehicles (UAVs) that works without Ground Control Points (GCP) is evaluated. The platform has a Direct Georeferencing (DG) module that includes a low-cost Global Positioning System (GPS). A flight test was performed to verify positioning accuracy in DG mode without using GCP. Preliminary results of a $300 \mathrm{~m}$ high flight show accuracy of $5 \mathrm{~m}$ in the horizontal direction, and $10 \mathrm{~m}$ vertically. It is concluded that the proposed platform is safe and economical to collect critical spatial information in case of urgent response for relief applications, and disaster assessment, where GCPs are not available [14].

The photogrammetric mapping work with a fixed-wing Q200 UAV, through the use of Precise Positioning of Points established with Global Positioning System (GPS-PPP), is a solution to space operation restrictions such as a) installation and use of terrestrial control points, b) use of local ter of referenced stations. flight missions, respecti to three centimeters in allowing large-scale challenging geographical locations [15].
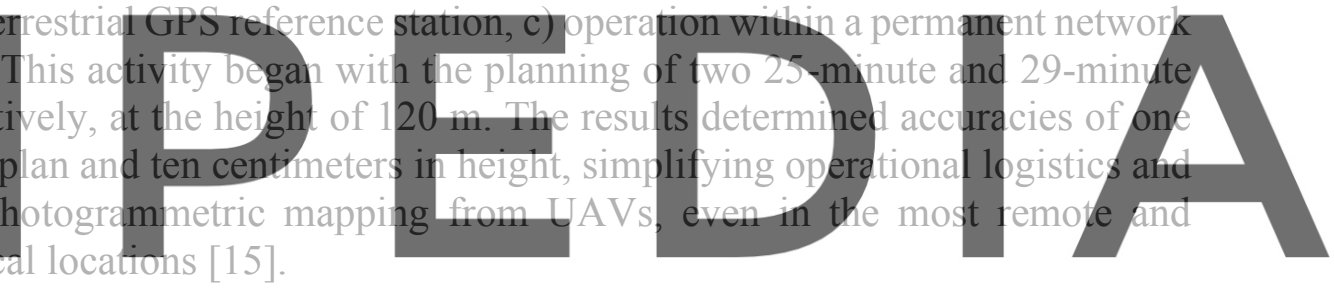

Register for freesatchttps//www.scipedia.com to download the version without the watermark

Establish a long-term structural health monitoring methodology for the historic building "Santa María de la Asunción" Cathedral using unmanned aerial vehicles, Global Position System, and ambient vibration tests.

\section{DESCRIPTION OF THE STRUCTURE UNDERSTUDY}

The "Santa María de la Asunción" Cathedral, located in the city of Chilpancingo; Guerrero, Mexico (coordinates $17^{\circ} 33^{\prime} 09.4$ "N $99^{\circ} 30^{\prime} 04.6^{\prime \prime} \mathrm{W}$ ), (Figure 2) was built in the mid-16th century in a neoclassical style, on an area of $1056 \mathrm{sqm}$ and $33.31 \mathrm{~m}$ of height. It has great historical value since it was the place where the First Anahuac Congress was held headed by General José María Morelos y Pavón, a patriot from New Spain and the architect of the Mexican independence movement. Figure 2 shows the current state of this historic building. 


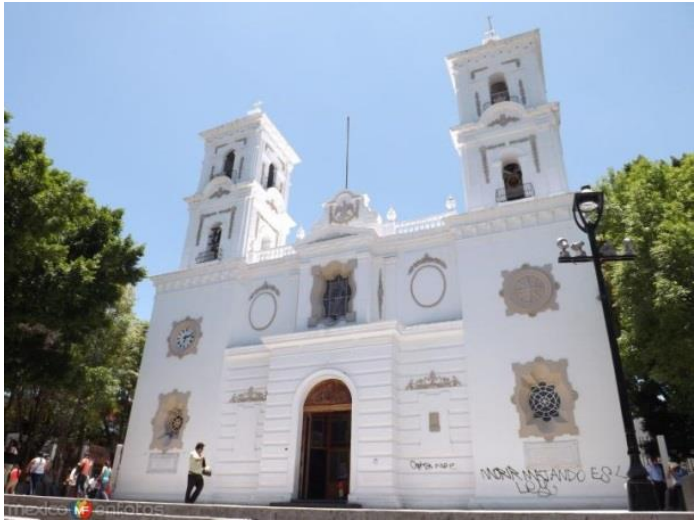

a)

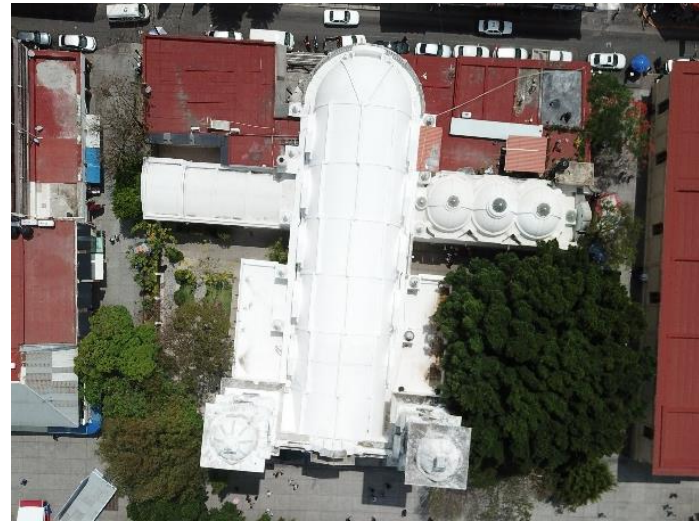

b)

Figure 2: "Santa María de la Asunción" Cathedral, a) Main façade, b) Top view

The original adobe-based structure partially collapsed and was demolished after the 1902 earthquake. Some years later, the construction began in the same place as the original temple. The left tower partially collapsed with the earthquake of 1957, and with the earthquake of 1985, the right tower suffered severe damage, so they were repaired after those events. Earthquake of December $2011(\mathrm{Mw}=6.5)$ caused cracks at the bases and the keystones of the arches of the

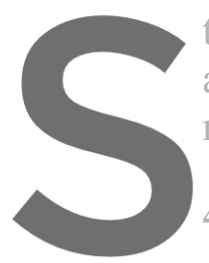
two bell towers, severe ones in the right tower, and also appeared in the frontispicce, nave vault [16].

\section{4}
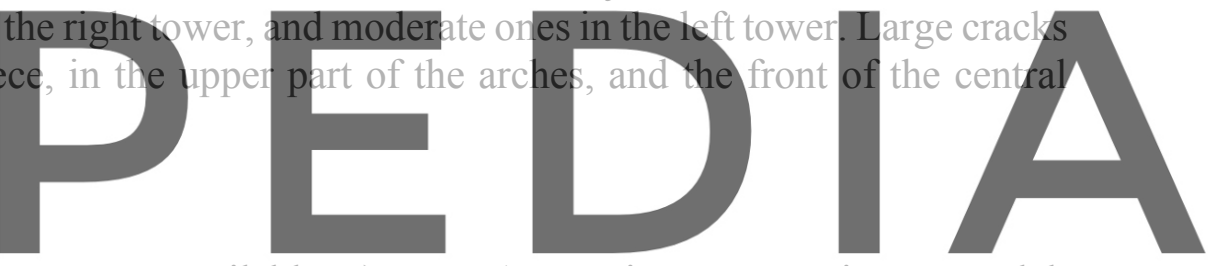

According to the technical resources available: a) DJI UAV equipment, Mavic Pro model,

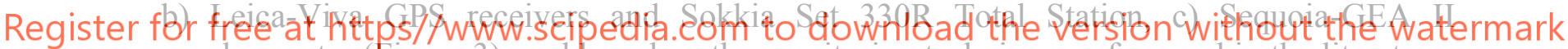
accelerometer (Figure 3); and based on the monitoring techniques referenced in the literature, the methodology described below has been defined (Figure 4).

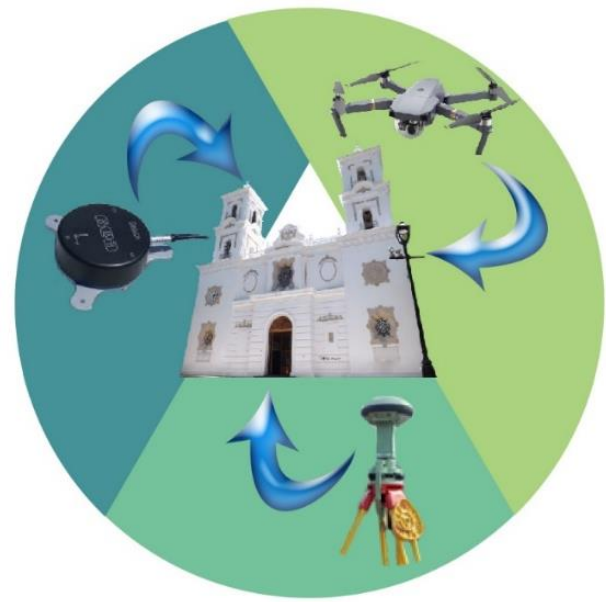

Figure 3: Scheme of integration for structural health monitoring 


\section{a) Establishment of azimuthal geodetic control line (ACL)}

The establishment of precise points (PPs) will be done with the GPS receivers and will be linked to the National Active Geodetic Network (NAGN). The geodetic survey will be carried out by the static method, following the Methodological guide established by the National Institute of Statistics and Geography of Mexico (INEGI), the minimum two-point junction defines the ACL.

\section{b) Establishment of the topographic support polygonal}

To set up the points of the polygonal from the PPs of the ACL, a Sokkia total station will be used, and the topographic survey will be performed by the point trilateration method. After, the coordinates must be adjusted by the method of least-squares to improve the accuracy.

\section{c) Establishment of Ground Control Points (GCPs)}

GCPs will be placed $50 \mathrm{~m}$ from the cathedral and will be measured the same way as PPs. GCPs will be used for horizontal adjustment of images taken by the UAV during the photogrammetric process.

d) Propagation of coordinates to the structure

The coordinates of the points of the topographic polygonal will be propagated to

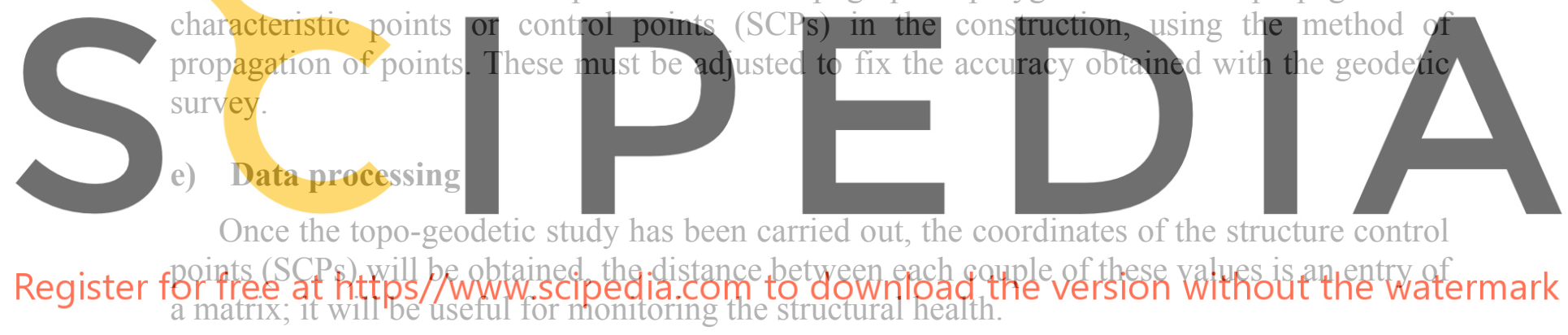

\section{f) Flight plan}

Revision of the UAV, batteries, drivers, control, calibration of the camera, and compass are necessary before the flight. It is planned to use the DJI GS Pro software, compatible with the UAV, where the parameters (area, height, speed, camera angle) will be defined.

\section{g) Image acquisition}

The acquisition of images in the field will be realized according to data of the flight plan. For greater accuracy, the take-off site of the UAV will be at one of the PP.

\section{h) Digital image processing}

Image processing will be achieved using Agisoft PhotoScan, photogrammetry software that allows image inspection to filter out those that do not fulfill the quality needed for processing. After filtering, if images are not enough for the processing, image acquisition will be repeated. 


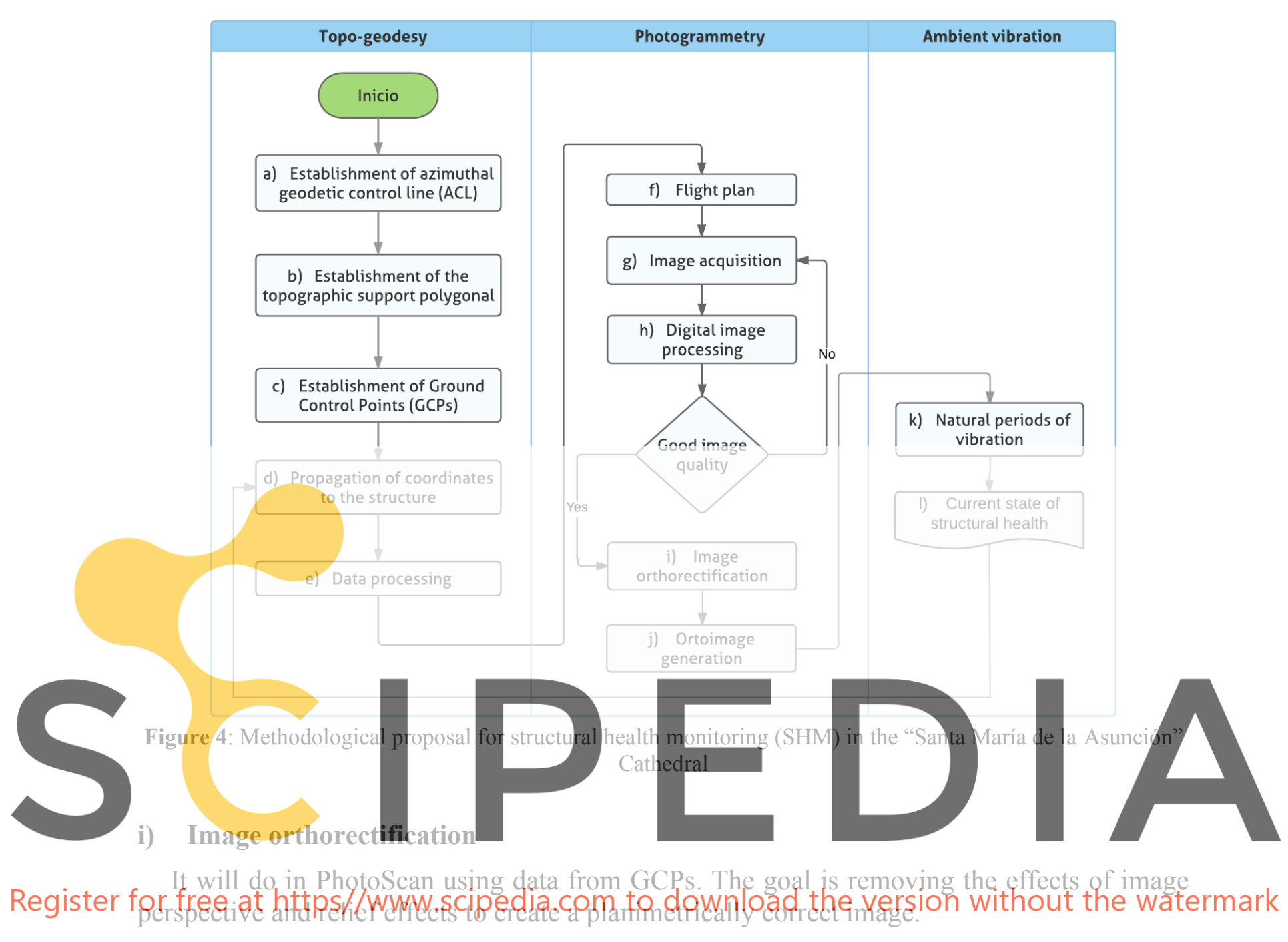

\section{j) Orthoimage generation:}

After the image orthorectification, a georeferenced orthoimage will be generated to identify the SCPs. The distance among the SCPs and the characteristic points of the construction in orthoimages will measure at different moments. Additionally, structural damage caused by cracks could be detected

\section{k) Natural periods of vibration}

A Sequoia-GEA II accelerometer will be used to measure the AV effect on the ground and structure. The obtained data will be processed by using two different identification methods: the Enhanced Frequency Domain Decomposition (EFDD) technique and the Stochastic Subspace Identification (SSI) method. EFDD is a frequency domain technique instead of SSI method, which is developed in the time domain. Modal Assurance Criterion between the EFDD and SSI results will be obtained for confirming the process [17]. 


\section{1) The current state of structural health}

At the end of the first cycle, an evaluation report of the existing structural health conditions of the "Santa María de la Asunción" Cathedral will be obtained. The comparison of measurement parameters of two consecutive cycles will define the change of structural level safety.

\section{CONCLUSIONS}

It is expected that the methodological proposal assesses the current state of the physical and dynamic conditions of the "Santa María de la Asunción" Cathedral. These data can help the authorities in making maintenance decisions and future monitoring of the structure. At the end of the project, the methodology can be qualified by making the necessary modifications. According to the architectural, structural characteristics, and use of the building, it is proposed to perform a monitoring every six months or after an earthquake with a seismic magnitude greater than six and epicenter less than $60 \mathrm{~km}$.

The combination of the three techniques (topo-geodesy, photogrammetry, and ambient vibration) was based on two reasons: a) the problems in historical constructions by repetitive seismic action, b) human and material resources. It would help if we remembered that in 2019, Mexico was affected by two major earthquakes, of which the effects were devastating on historical buildings near the epicenter.
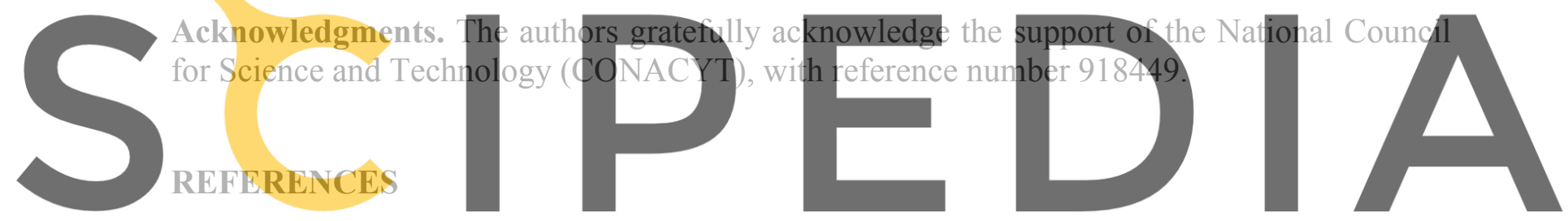

[1] K. Worden and J. M. Dulieu-Barton, "An Overview of Intelligent Fault Detection in

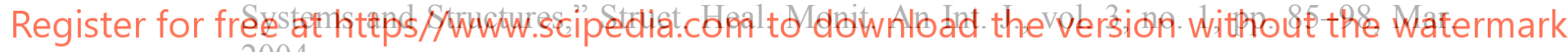
2004.

[2] B. Lei, N. Wang, P. Xu, and G. Song, "New Crack Detection Method for Bridge Inspection Using UAV Incorporating Image Processing," J. Aerosp. Eng., vol. 31, no. 5, p. 04018058 , Sep. 2018.

[3] S. B. Im, S. Hurlebaus, and Y. J. Kang, "Summary Review of GPS Technology for Structural Health Monitoring," J. Struct. Eng., vol. 139, no. 10, pp. 1653-1664, Oct. 2013.

[4] V. Gattulli, M. Lepidi, and F. Potenza, "Dynamic testing and health monitoring of historic and modern civil structures in Italy," Struct. Monit. Maint., vol. 3, no. 1, pp. 71-90, Mar. 2016.

[5] A. Guarnieri, N. Milan, and A. Vettore, "Monitoring of Complex Structure For Structural Control Using Terrestrial Laser Scanning (Tls) And Photogrammetry," Int. J. Archit. Herit., vol. 7, no. 1, pp. 54-67, Jan. 2013.

[6] F. Nex and F. Remondino, "UAV for 3D mapping applications: a review," Appl. Geomatics, vol. 6, no. 1, pp. 1-15, Mar. 2014.

[7] L. Duque, J. Seo, and J. Wacker, "Bridge Deterioration Quantification Protocol Using UAV," J. Bridg. Eng., vol. 23, no. 10, p. 04018080, Oct. 2018.

[8] A. Khaloo, D. Lattanzi, K. Cunningham, R. Dell'Andrea, and M. Riley, "Unmanned aerial 
vehicle inspection of the Placer River Trail Bridge through image-based 3D modeling," Struct. Infrastructure. Eng., vol. 14, no. 1, pp. 124-136, Jan. 2018.

[9] A. Murtiyoso and P. Grussenmeyer, "Documentation of heritage buildings using closerange UAV images: dense matching issues, comparison and case studies," Photogramm. Rec., vol. 32, no. 159, pp. 206-229, Sep. 2017.

[10] D. Germanese, G. Leone, D. Moroni, M. Pascali, and M. Tampucci, "Long-Term Monitoring of Crack Patterns in Historic Structures Using UAVs and Planar Markers: A Preliminary Study," J. Imaging, vol. 4, no. 8, p. 99, Aug. 2018.

[11] M. Gul and F. N. Catbas, "Ambient Vibration Data Analysis for Structural Identification and Global Condition Assessment," J. Eng. Mech., vol. 134, no. 8, pp. 650-662, Aug. 2008.

[12] A. Elyamani, O. Caselles, P. Roca, and F. Clapes, "Dynamic investigation of a large historical cathedral," Struct. Control Health Monit., vol 24, no. 3, pp. 1-25, May. 2016.

[13] C. Gentile, A. Ruccolo, and F. Canali, "Continuous Monitoring of the Milan Cathedral: dynamic characteristics and vibration-based SHM," Journal of Civil Structural Health Monitoring, vol. 9, pp. 671-688, Oct. 2019.

[14] K.-W. Chiang, M.-L. Tsai, and C.-H. Chu, "The Development of an UAV Borne Direct Georeferenced Photogrammetric Platform for Ground Control Point Free Applications," Sensors, vol. 12, no. 7, pp. 9161-9180, Jul. 2012.

[15] B. Grayson, N. T. Penna, J. P. Mills, and D. S. Grant, "GPS precise point positioning for UAV photogrammetry," Photogramm. Rec., vol. 33, no. 164, pp. 427-447, Dec. 2018.

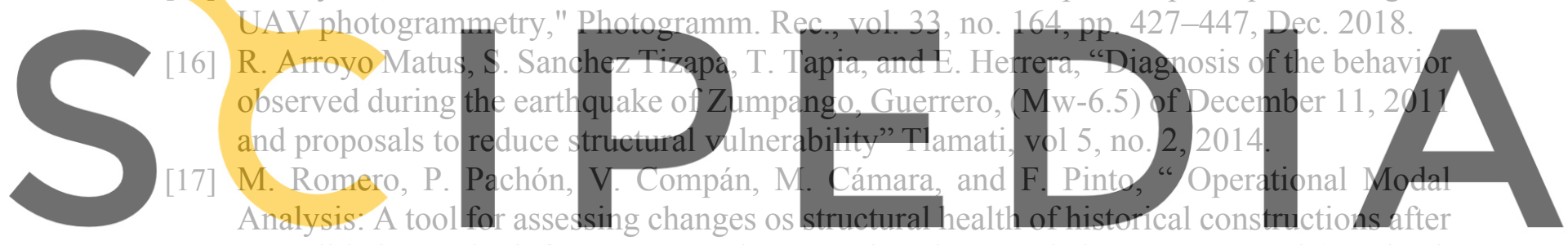
consolidation and reinforcement works-Jura Chapel (Jerez de la Frontera, Spain)," Shock 